\title{
Transtornos mentais comuns e o uso de práticas de medicina complementar e alternativa - estudo de base populacional
}

\author{
Commom mental disorders and the use of complementary \\ and alternative medicine - population - based survey
}

João Felício Rodrigues-Neto', Maria Fernanda Santos Figueiredo², Anderson Antônio de Silveira Fariaª Marise Fagundes ${ }^{4}$

\section{RESUMO}

Objetivo: Verificar a prevalência do transtorno mental comum (TMC) na população da cidade de Montes Claros, MG, e a existência de associação entre os fatores socioeconômicos e a utilização de práticas integrativas e complementares/medicina complementar e alternativa (PIC/MCA) com o TMC. Métodos: Estudo transversal. População alvo: moradores de Montes Claros. A amostragem foi probabilística, com 3.090 pessoas. Utilizouse formulário semi-estruturado e o self reporting questionnaire (SRQ-20) para identificação do TMC. Realizou-se regressão robusta de Poisson na análise com significância estatística considerada de $p<0,05$. Resultados: A prevalência de TMC foi de $23,2 \%$, sendo $75 \%$ usuários de PIC/MCA. Após controle para fatores de confusão, a prevalência de TMC foi maior naqueles com menor escolaridade ( $\mathrm{RP}=2,12$; IC $=1,80-2,49)$; com menor nível econômico $(R P=1,92 ; I C=1,07-3,44)$; com mais de 40 anos $(R P=1,30 ; I C=1,15-1,48)$; do gênero

\section{Palavras-chave}

Transtornos mentais, terapias complementares, terapias alternativas, homeopatia. feminino $(\mathrm{RP}=2,99$; $\mathrm{IC}=1,50-3,58)$ e mais freqüente entre os que recorreram à homeopatia ( $R P=1,52 ; \mathrm{IC}=1,12-2,08)$ e às benzedeiras $(R P=1,25 ; \mathrm{IC}=1,08-1,46)$. Conclusões: O TMC é muito freqüente na população de Montes Claros. As variáveis: escolaridade, nível econômico, idade e sexo, bem como a procura por homeopatia e benzedeiras estiveram associados ao TMC.

\begin{abstract}
Objective: To verify the Common Mental Disorders (CMD) prevalence in Montes Claros, and existence of association among social economic factors and the Complementary and Alternative Medicine (CAM) utilization with CMD. Methods: Cross-sectional study. Target population: Montes Claros residens. The sample was probabilistic with 3.090 people. A semi-structured questionnaire was used and the Self Reporting Questionnaire (SRQ-20) for identification of the CMD. It was
\end{abstract}

1 Departamento de Clínica Médica da Universidade Estadual de Montes Claros (Unimontes).

2 Departamento de Ciências da Saúde da Unimontes.

3 Departamento de Medicina da Unimontes.

4 Departamento de Ciências Exatas da Unimontes.

Financiamento: Conselho Nacional de Desenvolvimento Científico e Tecnológico (CNPq) no 403.426/04-q.

Endereço para correspondência: João Felício Rodrigues Neto

Av. Cula Mangabeira, 1562, Santo Expedito - 39401-002 - Montes Claros, MG

E-mail: joao.felicio@unimontes.br 


\section{Keywords}

Mental disorders, complementary therapies, alternative therapies, homeopathy. done Poisson regression in the analysis with significance statistics considered of $p<0,05$. Results: The prevalence of CMD was of $23,2 \%$, being $75 \%$ CAM users. The CMD prevalence was bigger in the those with minor schooling $(R P=2,12 ; I C=1,80-2,49)$; minor economic level $(R P=1,92 ; I C=$ $1,07-3,44)$; over 40 years $(R P=1,30 ; / C=1,15-1,48)$; in the women $(R P=2,99 ; / C=1,50-3,58)$. It was more frequent among who turn to homeopathy $(R P=1,52 ; I C=1,12-2,08)$ and spiritual healing by others ( $R P=1,25 ; I C=1,08-1,46)$. Conclusions: $T$ The $C M D$ is very frequent in population of Montes Claros. The variables: schooling, economic level; age; sex as well as turn to homeopathy and spiritual healing by others.
Os transtornos mentais atingem cerca de 450 milhões de pessoas no mundo, sendo resultante de múltiplas associações, tanto de fatores genéticos quanto de fatores comportamentais',2. A prevalência de transtornos mentais na população geral nos Estados Unidos atinge 48,6\% e na Turquia, abrange $12,2 \%^{3}$. No Brasil, a freqüência destes transtornos na população geral varia de $19 \%$ a 34\% $\%^{3,4}$.

Os transtornos mentais comuns (TMC) constituem sintomatologias depressivas e ansiosas que podem inclusive ter manifestações somáticas ${ }^{5}$, sendo questão de saúde pública ${ }^{6,7}$. A incidência dos TMC, como o transtorno depressivo, está aumentando, e possuem alta prevalência na população geral. No Brasil, a prevalência no âmbito da atenção primária atinge $40 \% 3$.

Alguns instrumentos podem ser utilizados para o rastreamento de TMC, como o general health questionnaire (GHQ-12) e o self reporting questionnaire (SRQ-20) ${ }^{8}$. No Brasil, este último tem sido o mais utilizado entre os estudos de rastreamento para $\mathrm{TMC}^{2,3,8,9}$.

Os fatores associados aos TMC, descritos tanto na literatura nacional quanto na internacional, são: escolaridade, idade, sexo, renda familiar e prática religiosa ${ }^{3,9-12}$. Alguns destes não podem ser modificados, como idade e sexo, enquanto os outros são passíveis de modificações, de maneira individual e/ou coletiva.

No Brasil, o campo das práticas integrativas e complementares (PIC) contempla sistemas médicos e recursos terapêuticos ${ }^{13}$. Em países em desenvolvimento, as PIC são denominadas medicina tradicional (MT), e medicina complementar e alternativa (MCA $)^{14}$. Neste trabalho, será utilizado o termo PIC/MCA para tratar de práticas integrativas e complementares/medicina complementar e alternativa. Apesar de a acupuntura e a homeopatia, no Brasil, serem especialidades médicas, não devendo ser denominadas alternativas, neste trabalho assim será feito, a fim de realizar comparações com estudos internacionais.

Em torno de dois terços da população mundial procuram cuidados para com a sua saúde na PIC/MCA ${ }^{15-17}$. A PIC/MCA consiste do conjunto de intervenções que não são amplamente discutidas em escolas médicas ou que geralmente não estão disponíveis nos hospitais ${ }^{16}$ ou que não estão em conformidade com os padrões da comunidade médica ${ }^{18}$. A utilização da PIC/MCA pode ser um fator que contribua para a redução dos TMC, já identificado em estudos realizados nos Estados Unidos ${ }^{16}$.

Esse trabalho se justifica pelo reduzido número de informações epidemiológicas de base populacional sobre TMC; a associação de fatores socioeconômicos e culturais que possam ser modificados, tanto em nível individual quanto coletivo; e a associação entre os TMC com as PIC/MCA, visando à busca de subsídios para políticas públicas de saúde para reduzir os TMC.

Os objetivos desse trabalho foram verificar a prevalência do transtorno mental comum na cidade de Montes Claros, norte de Minas Gerais, e identificar possível associação entre fatores socioeconômicos e culturais e a utilização da PIC/MCA com o TMC

\section{MÉTODOS}

\section{Desenho e população do estudo}

O estudo foi do tipo transversal com a população representada pelos residentes da cidade de Montes Claros, norte de Minas Gerais.

\section{Amostragem}

A amostra foi calculada com base na prevalência esperada de $20 \%$ de TMC. Trabalhando com erro alfa de $2 \%$ e beta de 90\% seriam necessárias cerca de 1.400 entrevistas para um estudo aleatório simples. Como o presente trabalho foi realizado por conglomerado, foi calculado o efeito do desenho da amostra ( $D E F=2,2)$, chegando-se à amostra total de 3.080 pessoas.

A amostra foi selecionada considerando os dados do Instituto Brasileiro de Geografia e Estatística (IBGE), que divide a região de Montes Claros em 216 setores censitários. Para a seleção da amostra não foram incluídos os setores não-urbanos e urbanos especiais (asilos, creches, orfanatos, hospitais, conventos), que totalizavam 35. Restaram 181 setores, sendo 163 "urbanos não-especiais" (onde reside cerca 
de 96\% da população urbana) e 18 "urbanos subnormais" favelas (cerca de $4 \%$ da população urbana).

Em uma segunda etapa, foi feito o sorteio para determinar a ordem dos domicílios a serem visitados dentro de cada setor, sendo sorteado o número três. Então, os domicílios residenciais foram amostrados em ordem de três, e o primeiro de cada três foi visitado. Considerando que cada setor censitário tinha em média de 200 a 300 domicílios (e um em cada três seria visitado), foram sorteados 35 setores, sendo 31 "urbanos não-especiais" (onde realizou-se 96\% das entrevistas) e quatro "urbanos subnormais" (onde realizou-se 4\% das entrevistas) para alcançar o tamanho da amostra calculada.

\section{Critérios de inclusão e exclusão}

Os critérios de inclusão utilizados foram: pessoas maiores de 18 anos, residentes em Montes Claros, de ambos os sexos. E foi utilizado como critério de exclusão não aceitar participar do estudo.

\section{Instrumentos e coleta de dados}

Foram aplicados formulários semi-estruturados (contendo 49 questões cada um), por cinco estudantes de graduação em saúde previamente treinados para a tarefa. Durante duas semanas foram realizadas entrevistas-piloto e os questionários revisados.

As entrevistas foram realizadas. Quando não era possível a entrevista, por não haver domicílio residencial ou por não haver morador, o domicílio seguinte era visitado e a contagem reiniciada deste ponto.

A cada semana os questionários eram recolhidos e verificados pelo coordenador de campo. Na hipótese de falha na coleta ou no preenchimento dos dados, o entrevistador era notificado e o questionário excluído.

Para avaliar as características socioeconômicas e culturais foram estudados: gênero, idade, cor, situação conjugal, religião, atividade econômica, escolaridade e renda familiar mensal. Para verificar a utilização de PIC/MCA foram analisados os sistemas médicos: acupuntura e homeopatia e recursos terapêuticos (massagem, programas de dietas/dietas populares, medicina ortomolecular, remédios populares, quiropraxia, exercícios físicos, benzedeiras, técnicas de relaxamento/meditação, grupos de auto-ajuda, oração a Deus com intenção terapêutica e guias espirituais/gurus/terreiros).

Para a identificação dos TMC foi utilizado o SRQ-20. O SRQ-20 é instrumento de rastreamento de transtornos mentais, validado para a população brasileira por Mari e Willians ${ }^{8}$. É composto por 20 perguntas de respostas "sim" ou "não". A pontuação é feita com contagem simples das respostas afirmativas, tendo mínimo de zero e máximo de 20 . O ponto de corte considerado foi a presença de sete respostas po- sitivas para identificar pessoas com TMC, com sensibilidade de $83 \%$ e especificidade de $80 \%{ }^{8}$.

\section{Análise estatística}

Os dados foram compilados e estruturados em um banco de dados, utilizando o programa estatístico Statístical Pocckage for the Social Sciences (SPSS) for Windows versão 15.0. As etapas da análise incluíram, respectivamente, descrição da amostra, análise bivariada das características socioeconômicas e culturais com TMC - teste do qui-quadrado, razões de prevalência (RP) e intervalos de confiança de 95\% (IC95\%) - e análise multivariada. A análise multivariada foi realizada por meio de regressão de Poisson com variância robusta, utilizando-se como variável dependente o TMC, e como variáveis independentes aquelas com nível de significância estatística do valor de $p \leq 0,20$ nas análises bivariadas. Ao final da análise, as variáveis com pelo menos uma categoria com valor de $\mathrm{p}<0,05$ no teste de Wald foram consideradas associações estatisticamente significantes com o desfecho.

O trabalho foi conduzido dentro dos padrões exigidos pela declaração de Helsink e aprovado pelo Comitê de Ética e Pesquisa (CEP) da Universidade Estadual de Montes Claros (Parecer no 129/2004). Antes do início da entrevista era realizada a leitura do termo de consentimento livre e esclarecido, o qual, em seguida, era assinado pelo entrevistado.

\section{RESULTADOS}

Foram aplicados 3.090 questionários, porquanto 1.810 $(58,6 \%)$ pessoas tinham até 8 anos de estudo; 1.618 $(52,4 \%)$ eram casadas e $1.937(62,7 \%)$ eram do sexo feminino (Tabela 1).

Na amostra, as pessoas com TMC, na comunidade da cidade de Montes Claros, totalizaram 716, com prevalência de $23,2 \%$. Entre os entrevistados com este transtorno, foi verificado que 395 (54,9\%) viviam com um companheiro; 709 (98,6\%) possuíam renda familiar mensal máxima de quatro salários mínimos e 595 (83,1\%) eram do sexo feminino (Tabela 1). Observou-se que entre o total de entrevistados com TMC, 452 (63\%) não trabalhavam, e entre os que trabalhavam $242(33,8 \%)$ referiam ao setor serviços, como o ramo de negócios de suas ocupações.

As pessoas com TMC utilizaram a PIC/MCA com maior freqüência que a população geral, 74,8\% e 70\%, respectivamente $(p=0,001)$. O uso das PIC/MCA variou entre aqueles com presença de TMC: entre um $(0,1 \%)$ usuário da medicina ortomolecular a $427(59,3 \%)$ que utilizaram oração a Deus como intento terapêutico (Tabela 1). Entre os 74 (2,4\%) usuários da homeopatia, o TMC esteve presente em 26 (35,1\%). 
Tabela 1. Características socioeconômicas e culturais e o uso de práticas integrativas e complementares/medicina complementar e alternativa (PIC/MCA) de 3.090 entrevistados, segundo a prevalência de transtornos mentais comuns (TMC) na cidade de Montes Claros, norte de Minas Gerais.

\begin{tabular}{|c|c|c|c|c|c|}
\hline \multirow[t]{2}{*}{ Variáveis } & \multicolumn{2}{|c|}{ Ausência de TMC } & \multicolumn{2}{|c|}{ Presença de TMC } & \multirow[t]{2}{*}{ p valor } \\
\hline & $\mathrm{N}$ & $\%$ & $\mathrm{~N}$ & $\%$ & \\
\hline \multicolumn{6}{|l|}{ Socioeconômicas e culturais } \\
\hline \multicolumn{6}{|l|}{ Cor } \\
\hline Branca & 1.073 & 45,3 & 301 & 41,7 & \multirow[t]{2}{*}{0,090} \\
\hline Não branca & 1.296 & 54,7 & 420 & 58,3 & \\
\hline \multicolumn{6}{|l|}{ Escolaridade } \\
\hline Oito ou mais anos de estudo & 1.105 & 46,6 & 175 & 24,3 & \multirow[t]{2}{*}{0,000} \\
\hline Até oito anos de estudo & 1.266 & 53,4 & 544 & 75,7 & \\
\hline \multicolumn{6}{|l|}{ Estado conjugal } \\
\hline Vive com um companheiro & 1.223 & 51,6 & 395 & 54,9 & \multirow{2}{*}{0,120} \\
\hline Vive sem um companheiro & 1.148 & 48,4 & 324 & 45,1 & \\
\hline \multicolumn{6}{|l|}{ Idade } \\
\hline Inferior a 40 anos & 1.408 & 59,4 & 310 & 43,0 & \multirow{2}{*}{0,000} \\
\hline 40 anos ou mais & 962 & 40,6 & 410 & 57,0 & \\
\hline Religião & & & & & \\
\hline Presença de prática religiosa & 2.279 & 96,1 & 687 & 95,5 & \\
\hline Ausência de prática religiosa & 92 & 3,9 & 32 & 4,5 & 0,492 \\
\hline Renda mensal familiar & & & & & \\
\hline Máximo de quatro salários mínimos & 2.260 & 95,3 & 709 & 98,6 & \\
\hline Maior que quatro salários mínimos & 111 & 4,7 & 10 & 1,4 & 0,000 \\
\hline Sexo & & & & & \\
\hline Masculino & 1.031 & 43,5 & 122 & 16,9 & \\
\hline Feminino & 1.339 & 56,5 & 598 & 83,1 & 0,000 \\
\hline Utilização de PIC/MCA & & & & & \\
\hline Sistemas médicos & & & & & \\
\hline Acupuntura & & & & & \\
\hline Não & 2.337 & 98,6 & 711 & 98,7 & \\
\hline Sim & 33 & 1,4 & 9 & 1,3 & 0,775 \\
\hline Homeopatia & & & & & \\
\hline Não & 2.323 & 98,0 & 694 & 96,4 & \\
\hline Sim & 47 & 2,0 & 26 & 3,6 & 0,009 \\
\hline Recursos terapêuticos & & & & & \\
\hline Benzedeiras & & & & & \\
\hline Não & 2.041 & 86,1 & 583 & 81,0 & \\
\hline Sim & 329 & 13,9 & 137 & 19,0 & 0,001 \\
\hline Exercícios físicos & & & & & \\
\hline Não & 1.737 & 73,3 & 565 & 78,5 & \\
\hline Sim & 633 & 26,7 & 155 & 21,5 & 0,006 \\
\hline Grupos de auto-ajuda & & & & & \\
\hline Não & 2.325 & 98,1 & 704 & 97,8 & \\
\hline Sim & 45 & 1,9 & 16 & 2,2 & 0,525 \\
\hline Guias espirituais, gurus, terreiros & & & & & \\
\hline Não & 2.318 & 97,8 & 695 & 96,5 & 0051 \\
\hline Sim & 52 & 2,2 & 25 & 3,5 & 0,051 \\
\hline Massagem & & & & & \\
\hline Não & 2.259 & 95,3 & 681 & 94,6 & 0415 \\
\hline Sim & 111 & 4,7 & 39 & 5,4 & 0,415 \\
\hline Medicina ortomolecular & & & & & \\
\hline Não & 2.365 & 99,8 & 719 & 99,9 & 0864 \\
\hline Sim & 5 & 0,2 & 1 & 0,1 & 0,864 \\
\hline Oração a Deus com intenção terapêutica & & & & & \\
\hline Não & 1.187 & 50,1 & 293 & 40,7 & م00 \\
\hline $\operatorname{Sim}$ & 1.183 & 49,9 & 427 & 59,3 & 0,000 \\
\hline Programas de dietas/dietas populares & & & & & \\
\hline Não & 2.214 & 93,4 & 657 & 91,2 & 0041 \\
\hline Sim & 156 & 6,6 & 63 & 8,8 & 0,041 \\
\hline Quiropraxia & & & & & \\
\hline Não & 2.334 & 98,5 & 705 & 97,9 & ח2 0 \\
\hline Sim & 36 & 1,5 & 15 & 2,1 & 0,280 \\
\hline Relaxamento/Meditação & & & & & \\
\hline Não & 2.299 & 97,0 & 704 & 97,8 & \\
\hline Sim & 71 & 3,0 & 16 & 2,2 & 0,311 \\
\hline Remédios populares & & & & & \\
\hline Não & 1.633 & 68,9 & 500 & 69,5 & 0784 \\
\hline Sim & 737 & 31,1 & 220 & 30,5 & $0, / 84$ \\
\hline
\end{tabular}


Entre todos os entrevistados e entre os usuários de PIC/MCA, 1.055 (34,1\%) e 833 (38,6\%), respectivamente, relataram ter algum problema de saúde. Entre os com TMC, 452 (63\%) mencionaram a presença de outra morbidade, sendo as mais relatadas: hipertensão (14,6\%), alterações na coluna vertebral e articulações (5,4\%), cardiopatia (4,6\%), gastrite $(4,4 \%)$, diabetes melito $(3,3 \%)$ e depressão (2,4\%). Destaca-se que $413(91,4 \%)$ desses relataram estar em tratamento ou já terem sido tratados com a medicina convencional. Entretanto, 147 (20,5\%) estão insatisfeitos ou pouco satisfeitos com esta forma terapêutica.

Quando as variáveis socioeconômicas das pessoas com TMC foram submetidas à análise bivariada, para obtenção das medidas de associação com $p<0,20$, observou-se que houve as seguintes associações: cor $(p=0,090)$, escolaridade $(p=0,000)$, estado conjugal $(p=0,120)$, idade $(p=0,000)$, renda familiar mensal $(p=0,000)$ e sexo $(p=0,000)$. Não houve associação estatística entre a variável religião ( $p=$ 0,492) e o TMC. Em relação, ao uso da PIC/MCA, foi verificada a associação deste distúrbio com as seguintes terapias alternativas: sistemas médicos/benzedeiras ( $p=0,001)$, recursos terapêuticos: exercícios físicos ( $p=0,006)$, guias espirituais, gurus, terreiros $(p=0,051)$, homeopatia $(p=0,009)$, oração a Deus $(p=0,000)$ e programas dietas/dietas populares ( $p$ $=0,041)$. Não houve associação estatística entre o TMC e as seguintes formas terapêuticas: acupuntura ( $p=0,775)$, grupos de auto-ajuda ( $p=0,525)$, massagem $(p=0,415)$, medicina ortomolecular $(p=0,864)$, quiropraxia $(p=0,280)$, relaxamento/meditação $(p=0,311)$ e remédios populares ( $p$ $=0,784)$ (Tabela 1).

A análise multivariada, realizada com as variáveis que apresentaram $p<0,20$ na análise bivariada, mostrou, em relação às variáveis socioeconômicas, que a prevalência do TMC é maior entre os indivíduos com menor escolaridade, considerado até oito anos de estudo $(R P=2,12 ; \mathrm{IC}=1,80$ 249); entre aqueles com idade a partir de 40 anos ( $R P=1,30$; IC = 1,15-1,48); naqueles com renda familiar mensal máxima de quatro salários mínimos ( $\mathrm{RP}=1,92$; $\mathrm{IC}=1,07-3,44)$; e nas mulheres (RP = 2,99; IC = 2,50-3,58). Quanto à utilização de PIC/MCA, verificou-se maior prevalência de TMC nos indivíduos que usavam o sistema médico: a homeopatia (RP = 1,52 ; IC $=1,12-2,08$ ) e o recurso terapêutico: as benzedeiras $(\mathrm{RP}=1,25 ; \mathrm{IC}=1,08-1,46)$ (Tabela 2).

\section{DISCUSSÃO}

Os resultados deste estudo foram obtidos a partir de amostra representativa da população de adultos, homens e muIheres, de uma cidade de porte médio do sudeste do Brasil. O desfecho foi avaliado por meio do TMC que, embora seja medido com instrumento de rastreamento, como o SRQ-20, apresenta possibilidades de falso-positivo.
Tabela 2. Análise multivariada entre as variáveis socioeconômicas, utilização de práticas integrativas e complementares/medicina complementar e alternativa (PIC/MCA) e transtornos mentais comuns (TMC).

\begin{tabular}{|c|c|c|c|}
\hline \multirow[t]{2}{*}{ Variáveis } & \multirow[t]{2}{*}{$\mathrm{RP}$} & \multicolumn{2}{|c|}{ IC95\% } \\
\hline & & Inferior & Superior \\
\hline \multicolumn{4}{|l|}{ Características socioeconômicas } \\
\hline \multicolumn{4}{|l|}{ Escolaridade } \\
\hline Oito ou mais anos de estudo & 1,00 & & \\
\hline Até oito anos de estudo & 2,12 & 1,80 & 2,49 \\
\hline \multicolumn{4}{|l|}{ Idade } \\
\hline Inferior a 40 anos & 1,00 & & \\
\hline 40 anos ou mais & 1,30 & 1,15 & 1,48 \\
\hline \multicolumn{4}{|l|}{ Renda mensal familiar } \\
\hline Máximo de quatro salários mínimos & 1,00 & & \\
\hline Maior que quatro salários mínimos & 1,92 & 1,07 & 3,44 \\
\hline \multicolumn{4}{|l|}{ Sexo } \\
\hline Masculino & 1,00 & & \\
\hline Feminino & 2,99 & 2,50 & 3,58 \\
\hline \multicolumn{4}{|l|}{ Utilização de PIC/MCA } \\
\hline \multicolumn{4}{|l|}{ Sistemas médicos } \\
\hline \multicolumn{4}{|l|}{ Homeopatia } \\
\hline Não utiliza homeopatia & 1,00 & & \\
\hline Utiliza homeopatia & 1,52 & 1,12 & 2,08 \\
\hline \multicolumn{4}{|l|}{ Recursos terapêuticos } \\
\hline \multicolumn{4}{|l|}{ Benzedeiras } \\
\hline Não procura por benzedeiras & 1,00 & & \\
\hline Procura por benzedeiras & 1,25 & 1,08 & 1,46 \\
\hline
\end{tabular}

Outra limitação potencial dos resultados deste estudo seria a causalidade reversa, freqüente na interpretação de estudos transversais. Esse problema espera-se ter sido amenizado com os modelos de análise multivariada definidos a priori. Contudo, os resultados estão sendo interpretados com muita prudência, tendo-se em vista a possibilidade desse viés.

Os TMC produzem impacto considerável e amplo na vida do indivíduo e dos familiares envolvidos, já que constituem importante causa de incapacidade ${ }^{2}$ e prejuízos importantes nas funções social e física ${ }^{6,19}$.

$\mathrm{Na}$ cidade de Montes Claros, a prevalência dos TMC não foi muito diferente do encontrado em outros estudos, variando de $18,5 \%$ a $28,5 \%$ no Brasil $^{2,3}$ e de $4,26 \%$ a $33,6 \%$ internacionalmente ${ }^{10,11}$. Em estudos realizados na cidade de Pelotas, RS, observou-se que a prevalência foi de $28,5 \%$ na comunidade ${ }^{3}, 18,5 \%$ em estudantes dos cursos de direito e medicina ${ }^{2}$, e $21,1 \%{ }^{12}$, entre os estudantes de psicologia. Em outro estudo, realizado em São Paulo, entre motoristas e cobradores de ônibus, a prevalência foi de 20,3\% ${ }^{5}$. Em outros países a prevalência desse transtorno foi de $33,6 \%$ no Paquistão ${ }^{10}, 16,8 \%$ em Cingapura ${ }^{20}$ e 4,26\% em Taiwan" . $^{11}$ Observou-se que não houve grande variação nos estudos brasileiros, o mesmo não ocorrendo nos estudos externos. Ressalta-se, ainda, que talvez a prevalência dos TMC encontrada neste trabalho pode estar superestimada, já que na análise multivariada observou-se que os TMC são mais 
freqüentes em pessoas com renda de, no máximo, quatro salários mínimos e mais de 40 anos de idade, e na população brasileira a freqüência de pessoas nesta faixa etária foi menor e com aquela renda, maior, quando comparadas a esta amostra.

Não foram observadas diferenças com TMC entre pessoas brancas e não-brancas e entre aqueles que vivem com ou sem um companheiro.

A freqüência do TMC maior em pessoas com menor escolaridade foi vista também internacionalmente, como no Paquistão ${ }^{10}$. Gazalle et al. ${ }^{9}$ afirmam que a maior escolaridade mostra-se como fator protetor importante para a ocorrência deste distúrbio, justificando ações com o intuito de promover melhora na escolaridade das pessoas.

O TMC mais freqüente em pessoas com mais idade também foi notado no Rio Grande do Sul, por Costa et al. ${ }^{3}$, e em outros países, por Chien et al.11 em Taiwan e por Mirza e Jenkins ${ }^{10}$ no Paquistão. A idade é um fator não modificável, entretanto a idade maior que 40 anos pode estar relacionada a condições comuns do processo de envelhecimento, como maior exposição a doenças crônicas não-transmissíveis, dificuldade de reiniciar projetos de vida, conseguir novo emprego e novos relacionamentos estáveis ${ }^{3}$. Chien et al. ${ }^{11}$ ainda destacam a necessidade do cuidado com a saúde mental dos idosos. Entretanto, o estudo de Souza e Silva ${ }^{5}$ sugere que o fato de ser jovem constitui fator de risco para o TMC.

A correlação entre a renda familiar e a presença de TMC também foi observada em outras regiões do Brasil, como São Paulo, SP5, Zona da Mata de Pernambuco, PE7, e Pelotas, RS $^{3}$, e em outros países, como Taiwan'1", Paquistão ${ }^{10}$ e Inglaterra ${ }^{21}$. Chien et al. ${ }^{10}$ e Mirza e Jenkins ${ }^{11}$ sugerem que esta associação de maior freqüência de TMC em pessoas com menor nível econômico deve-se ao fato de elas experimentarem mais situações de estresse psicossocial, sendo importante a realização de atividades de promoção em saúde mental, além de ações governamentais de melhora das condições de vida para as pessoas.

$\mathrm{O}$ achado de TMC ser mais freqüente em mulheres é especialmente relevante no estudo de base populacional, porque refuta a idéia de que o TMC seja mais diagnosticado em mulheres em virtude de elas procurarem mais os serviços de saúde 9 . Não obstante o fator sexo também não poder ser modificável, o sexo feminino pode apresentar maior vulnerabilidade na ocorrência de TMC, por causa de várias diferenças nos aspectos biopsicosociais em relação aos homens, como alterações hormonais, variáveis sociais (gestação, jornada de trabalho, estado conjugal e número de filhos) e o modo como as mulheres estabelecem suas relações nas diversas culturas e no tempo 6,11,22. Tanto no Brasil $^{3,7,19}$ quanto em outros países, como Taiwan"1 e Paquistão ${ }^{10}$, foi observada maior ocorrência dos TMC em mulheres. Esses distúrbios consistem no terceiro problema de saú- de em mulheres nos países desenvolvidos e no quinto em países subdesenvolvidos ${ }^{19}$

A PIC/MCA foi opção terapêutica significativa para as pessoas com TMC neste trabalho, sendo utilizada por 38\% quando a oração a Deus não é incluída, e por 74,8\%, quando esta é acrescentada. Nos Estados Unidos, foi observado que 40,9\% da população geral utilizaram pelo menos um tipo de terapia alternativa, não incluindo a oração a Deus, e em relação ao tratamento de TMC, a técnica de relaxamento e cura espiritual foram as terapias alternativas mais utilizadas $^{16}$

Entre os sistemas médicos de PIC/MCA, verificou-se que a prevalência de uso da homeopatia é maior entre as pessoas com TMC, o que pode estar relacionado ao fato de essa forma terapêutica ter sido reconhecida pelo Conselho Federal de Medicina (CFM) há mais tempo, quando comparada às outras formas, como a acupuntura. Ademais, a homeopatia oferece menor custo e maior satisfação, quando comparada com a utilização de psicofármacos ou psicoterapias $^{23}$ e ainda menor presença de efeitos secundários ${ }^{17}$.

Entre os recursos terapêuticos, a procura por benzedeiras pode ser explicada por ser prática instaurada histórica e culturalmente aceita no Brasil, podendo ser a única opção para aqueles que não têm acesso regular aos serviços de saúde. Moreira-Almeida et al. ${ }^{24}$ destacam que as pessoas oram mais quando estão em situações de estresse ou doentes.

Ressalta-se, também, que a utilização destas formas terapêuticas pode estar relacionada, como observado nesse estudo, ao fato de que muitos estão insatisfeitos ou pouco satisfeitos com a medicina convencional e, ainda, ao estigma que permeia o transtorno mental, havendo preconceito por parte das pessoas em procurar atenção psiquiátrica. Atualmente, as pessoas com problemas de saúde mental podem estar buscando apoio no Centro de Atenção Psicossocial (CAPS), que consiste na porta de entrada da rede de serviços para as ações relativas à saúde mental, orientado pela Portaria no 224, do Ministério da Saúde, de 29 de janeiro de 1992, oferecendo atendimento de cuidados intermediários entre o regime ambulatorial e a internação hospitalar ${ }^{25}$. Entretanto, trata-se de serviço que ainda pode não ser do conhecimento de todos, o que limita a sua utilização.

\section{CONCLUSÃO}

Esse estudo possibilitou verificar que o TMC é muito freqüente na cidade de Montes Claros. Os fatores associados aos TMC identificados neste e em outros estudos evidenciaram que alguns não podem ser modificados, enquanto outros são passíveis de modificações, de maneira individual ou coletiva. Os fatores não-modificáveis são sexo e idade. O fator utilização das PIC/MCA permite ações individuais 
capazes de reduzir a freqüência dos TMC na comunidade. Já os fatores renda e escolaridade necessitam também de ações coletivas, na medida em que o crescimento socioeconômico do país poderia oferecer melhores oportunidades às pessoas, entre elas o acesso à PIC/MCA, melhores níveis de escolaridade e renda.

\section{AGRADECIMENTOS}

Os autores agradecem ao Conselho Nacional de Desenvolvimento Científico e Tecnológico (CNPq) pelo apoio financeiro prestado para realização deste trabalho.

\section{REFERÊNCIAS}

1. WHO (World Health Organization). Division of mental health and prevention of substance abuse. WHOQOL and spirituality, religiousness and personal beliefs (SRPB). Genebra; 1998 (Report on WHO consultation).

2. Volcan SMA, Sousa PLR, Mari JJ, Horta BL. Relaçãa entre bem-estar espiritual e transtornos psiquiátricos menores: estudo transversal. Rev Saude Publica. 2003;37(4).

3. Costa JSD, Menezes AMB, Olinto MTA, Gigante DP, Macedo S, Britto MAP, et al. Prevalência de distúrbios psiquiátricos menores na cidade de Pelotas, RS. Rev Bras Epidemiol. 2002;5(2).

4. Almeida-Filho N, Mari J, Coutinho E, Franca JF, Fernandes J, Andreoli SB, et al. Brazilian multicentric study of psychiatric morbidity. Methodological features and prevalence estimates. Br J Psychiatry. 1997;171:524-9.

5. Souza MFM, Silva GR. Risco de distúrbios psiquiátricos menores em área metropolitana na região Sudeste do Brasil. Rev Saude Publica. 1998;32(1).

6. Fleck MPA, Lima AFBS, Louzada S, Schestasky G, Henriques A, Borges VR, et al. Associação entre sintomas depressivos e funcionamento social em cuidados primários à saúde. Rev Saude Publica. 2002;36(4).

7. Costa AG, Ludemir AB. Transtornos mentais comuns e apoio social: estudo em comunidade rural da Zona da Mata de Pernambuco, Brasil. Cad Saude Publica. 2005;21(1).

8. Mari J, Willians P. A validity study of a psychiatric screening questionnaire (SRQ-20) in primary care in the city of São Paulo. Br J Psychiatry. 1986;148:23-6.
9. Gazalle FK, Lima MS, Tavares BF, Halla PC. Sintomas depressivos e fatores associados em população idosa no Sul do Brasil. Rev Saude Publica. 2004;38(3).

10. Mirza I, Jenkins R. Risk factors, prevalence, and treatment of anxiety and depressive disorders in Pakistan: systematic review. BMJ. 2004;328:794.

11. Chien I-Chia, Chou Yiing-Jeng, Lin Ching-Heng, Bih Shin-Huey, Chou Pesus. Prevalence of Psychiatric Disorders Among National Health Insurance Enrollees in Taiwan. Psychiatric Services. 2004;55(6) [acesso em 2007 Mar 12]. Disponível em: http://www. ps.psychiatryonline.org.

12. Gastaud MB, Souza LDM, Braga L, Horta CL, Oliveira FM, Sousa PLR, et al. Bem-estar espiritual e transtornos psiquiátricos menores em estudantes de psicologia: estudo transversal. Rev Psiquiatr Rio Gd Sul. 2006;28(1).

13. Brasil. Portaria no 971, 3 de maio de 2006. Ministério da Saúde; 2006

14. OMS (Organizaçión de la Salud Mundial). Estrategia de la OMS sobre medicina tradicional 2002-2005. Genebra; 2002

15. Eisemberg DM. Advising patients who seek alternative medical therapies. Ann Intern Med. 1997;127:61-9.

16. Eisemberg DM, Davis RB, Ettner SL, Scott A, Wilker S, Rompay MV, et al. Trends in Alternative Medicine use in the United States, 1990-1997: results of a follow-up national survey. JAMA. 1998:280:1569-75.

17. Pal SK. Complementary and alternative medicine: an overview. Current Science. 2002:82(5):518-24

18. Gevitz N. Three perspectives on unorthodox medicine. In: Gevitz N, editor. Other healers: unorthodox medicine in America. Baltimore, John Hopkins University Press; 1998, p. $1-28$

19. Lopes CS, Faerstein E, Chor D. Eventos de vida produtores de estresse e transtornos mentais comuns: resultados do estudo pró-saúde. Cad Saude Publica. 2003;19(6).

20. Fones CSL, Kua EH, Ng TP, Ko SM. Studying the Mental Health of a Nation - a preliminary report on a population survey in Singapore. SMJ. 1995.

21. Wiggins RD, Schofield P, Sacker A, Head J, Bartley M. Social position and minor psychiatric morbidity over time in the British Household Panel Survey 1991-1998. J Epidemiol Community Health. 2004;58:779-87.

22. Justo LP, Calil HM. Depressão - o mesmo acometimento para homens e mulheres? Rev Psiquiatr Clin. 2006;33(2).

23. Bell IR. Depression research in homeopathy: Hopeless or hopefull? Guest editorial. Homeopathy. 2005:94:141-4.

24. Moreira-Almeida A, Lotufo Neto F, Evangelista J. Religiousness and mental health: a review. Rev Bras Psiquiatr. 2006.

25. Brasil. Portaria nº 224, de 29 de janeiro de 1992. Ministério da Saúde; 1992. 